Questions vives

\section{Questions Vives}

Recherches en éducation

Vol. $6 n^{\circ} 18$ | 2012

Les recherches sur les pratiques enseignantes efficaces

\title{
Les pratiques pédagogiques des enseignants universitaires : Quelle variété pour quelle efficacité ?
}

Teaching practices of the University Professors: Which Variety for which

effectiveness?

\section{Amélie Duguet et Sophie Morlaix}

\section{(2) OpenEdition}

Journals

\section{Édition électronique}

URL : http://journals.openedition.org/questionsvives/1178

DOI : $10.4000 / q u e s t i o n s v i v e s .1178$

ISBN : 978-2-8218-1394-6

ISSN : $1775-433 \mathrm{X}$

Éditeur

Université Aix-Marseille (AMU)

Édition imprimée

Date de publication : 15 décembre 2012

Pagination : $93-110$

ISSN : 1635-4079

Référence électronique

Amélie Duguet et Sophie Morlaix, «Les pratiques pédagogiques des enseignants universitaires : Quelle variété pour quelle efficacité ?», Questions Vives [En ligne], Vol.6 n¹8 | 2012, mis en ligne le 15 octobre 2013, consulté le 10 décembre 2020. URL : http://journals.openedition.org/questionsvives/ 1178 ; DOI : https://doi.org/10.4000/questionsvives.1178

Ce document a été généré automatiquement le 10 décembre 2020.

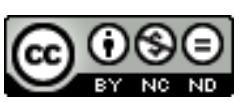

Questions Vives est mis à disposition selon les termes de la licence Creative Commons Attribution Pas d'Utilisation Commerciale - Pas de Modification 4.0 International. 


\title{
Les pratiques pédagogiques des enseignants universitaires: Quelle variété pour quelle efficacité?
}

\author{
Teaching practices of the University Professors: Which Variety for which \\ effectiveness?
}

Amélie Duguet et Sophie Morlaix

\section{Introduction}

1 En France, d'après le ministère de l'enseignement supérieur et de la recherche, près de $80 \%$ des nouveaux bacheliers se sont inscrits dans l'enseignement supérieur en 2010. Ainsi, plus d'un étudiant sur deux, titulaire d'un bac général, a fait le choix de poursuivre ses études à l'université. Le nombre de bacheliers technologiques et professionnels poursuivant leurs études à l'université est également en constante augmentation (respectivement 18,7 \% et 7,9\% en 2010). Les raisons de cet engouement pour l'université tiennent en grande partie au fait qu'elle s'est largement démocratisée ces dernières décennies, même si cette démocratisation apparait pour certains chercheurs (Prost, 1986) plus quantitative que qualitative. Mais elle n'en demeure pas moins conséquente puisque si l'université comptait 215000 étudiants en son sein en 1960, ils sont 1312000 en 2005 (Vasconcellos, 2006). Cette «massification» des effectifs, terme fréquemment employé par des chercheurs tels que Lévy-Garboua (1976), Jarousse (1984), Galland et Oberti (1996), Romainville (2000), ou encore Beaud (2002) pour décrire la multiplicité et l'hétérogénéité du public étudiant, a progressivement contribué au développement de nombre de recherches, visant à analyser les phénomènes ayant cours à l'université et impactant sur la réussite des étudiants. C'est justement dans ce cadre que s'inscrivent les travaux portant sur la pédagogie universitaire, et plus précisément sur les pratiques pédagogiques des enseignants exerçant à l'université. Ainsi cette thématique a-t-elle été abordée sous différents aspects par des chercheurs de diverses nationalités. L'objectif de cet article 
sera, dans un premier temps, de faire le point sur les travaux portant sur les pratiques pédagogiques des enseignants à l'université ${ }^{1}$. Cette revue de différentes recherches menées sur le sujet nous conduira à nous interroger sur les effets induits par ces pratiques sur les étudiants de première année. Enfin, sera évoquée une perspective de recherche novatrice, faisant l'objet d'une thèse de doctorat en cours de rédaction, et portant sur la relation entre les pratiques pédagogiques des enseignants d'une part, et la motivation ainsi que les manières d'étudier des étudiants de première année universitaire d'autre part, ces facteurs constituant, sans doute parmi d'autres, des indicateurs de l'efficacité enseignante.

\section{De l'analyse des travaux sur la description des pratiques pédagogiques dans le supérieur}

2 La pédagogie est une notion couramment abordée dans les écrits des chercheurs en sciences de l'éducation. Le vocabulaire relatif à ce domaine est conséquent. Cette notion regroupe en effet un grand champ lexical, comprenant notamment le terme de " pratiques pédagogiques ». Or, ce terme, bien que couramment usité dans la littérature scientifique, fait bien souvent l'objet d'un amalgame avec d'autres locutions, telles celles de "méthodes pédagogiques » ou "modèles pédagogiques ». Ainsi, d'après Morandi et La Borderie (1998), le modèle pédagogique est le «principe conducteur » d'une activité, les méthodes étant «leur mode de réalisation». En revanche, la pratique est considérée par Clanet (2001) comme étant le "fruit d'une interactivité entre des dimensions relevant des situations, des sujets et des processus ». Bru (2006) complète cette définition en ajoutant que lorsqu'elle est qualifiée de pédagogique, la pratique consiste à « mettre en place un certain nombre de conditions cognitives, matérielles, relationnelles, temporelles auxquelles les élèves sont confrontés». Ce dernier avertit cependant quant au fait que la pratique n'est finalement que rarement véritablement l'application d'une méthode, car « dans le cours de la pratique interviennent des évènements imprévus, des charges psychologiques, des contraintes, des exigences simultanées et parfois contradictoires qui font que la pratique ne suit pas toujours un chemin balisé».

3 Au-delà de ces problèmes de définitions, les pratiques des enseignants sont à l'origine d'un certain nombre de questionnements. Les recherches de Bru (2001) portant sur le premier cycle universitaire pointent notamment du doigt une "absence de connaissances suffisantes et détaillées » en ce qui concerne les pratiques enseignantes, rendant ainsi « difficile des travaux dont nul ne peut nier l'importance ». Clanet (2001) a tenté d'élargir l'assise de savoirs dont les scientifiques disposent en la matière. Ainsi, a-t-il pour objectif d'identifier la nature des «organisateurs des pratiques enseignantes en première année universitaire ». En s'appuyant sur un échantillon de 1818 étudiants de L1 inscrits dans les filières AES, psychologie et SVT sur les sites de Dijon, Nantes et Toulouse, il tente de véritablement décrire les pratiques des enseignants, cela en distinguant les pratiques déclarées des pratiques constatées, et montre qu'il existe une réelle variété des pratiques enseignantes au premier cycle, même si l'exposé oral de l'enseignant durant son intervention domine largement.

4 Même si les chercheurs ont abordé le thème des pratiques pédagogiques des enseignants sous divers angles, cela n'empêche pas nombre d'entre eux de regretter un manque de recherches portant sur le sujet. Ainsi, dans un rapport de recherche datant de 1999, 
Trinquier, Clanet et Alava se sont eux aussi intéressés aux pratiques déclarées et aux pratiques effectives des enseignants. Cependant, il leur a été permis de constater que " parler de pédagogie à l'université renvoie très souvent à s'intéresser aux dispositifs organisationnels", et non véritablement aux pratiques pédagogiques employées par les enseignants face aux étudiants. Dans un article encore plus récent, Adangnikou (2008) mentionne le fait qu'il n'existe finalement que très peu de recherches portant véritablement sur les pratiques d'enseignement à l'université.

\subsection{Des pratiques pédagogiques variées, peu adaptées à l'hétérogénéité du public}

5 Dès leur entrée à l'université, les étudiants subissent, en raison des différents «styles pédagogiques » auxquels ils sont confrontés, ce que Coulon (2005) nomme une «rupture psycho-pédagogique». Les travaux portant sur les pratiques enseignantes à l'université montrent justement la difficulté des étudiants à suivre les cours magistraux. Boyer et Coridian (2002) remarquent par exemple certains points de convergence entre les différents cours magistraux observés, ceux-ci demeurant dans la majorité des cas des " conférences monologues" ou des "monologues expressifs", face auxquels les étudiants rencontrent nombre de difficultés (Altet, 1994).

6 Or, ce modèle pédagogique dit « traditionnel » et apparemment généralement adopté par les enseignants durant les cours magistraux a fait l'objet de diverses critiques, certains chercheurs l'estimant «inadéquat » (Bireaud, 1990), voire même «inadapté» (Felouzis, 2003). Déjà en 1964, Bourdieu et Passeron expliquaient que c'était par «sa pédagogie de l'absence de pédagogie " que l'université permettait la reproduction de la hiérarchie des classes. Pour Bireaud (1990), le modèle traditionnel qui devait en théorie servir à assurer la reproduction de la communauté scientifique s'est dégradé et est de plus en plus inadapté. Celle-ci a par ailleurs tenté de mettre en avant les nouvelles méthodes pédagogiques qui pourraient être employées par les enseignants, comme la pédagogie par objectifs ou la pédagogie de projet. Mais elle n'est pas la seule à évoquer l'inadaptation des pratiques enseignantes.

7 Felouzis (2003) mentionne aussi le fait que le modèle pédagogique universitaire est inadapté aux nouveaux publics étudiants: ceci constitue selon lui «l'élément le plus pertinent expliquant la fuite des étudiants » de premier cycle. Pour Galinon-Mélénec (1996), il existe une réelle inadéquation entre «le besoin de la majorité des étudiants qui arrivent à l'université et l'offre d'enseignement faite par le corps des enseignants chercheurs». Coulon (2005) remarque d'ailleurs que les étudiants peinent à comprendre, ce au moins durant les premières semaines, les consignes non explicites qui leur sont données par les enseignants. Or, l'hétérogénéité croissante des étudiants entrant dans l'enseignement supérieur réclame des méthodes pédagogiques appropriées (Peretti, 2009).

8 Altet (2004) a justement cherché à savoir comment les enseignants disaient « prendre en compte les nouveaux publics et l'hétérogénéité perçue ». C'est ainsi qu'elle évoque la «mètis enseignante ", sorte de "bricolages adaptatifs » mis en place quotidiennement par les enseignants pour " gérer et gommer l'hétérogénéité des étudiants ».

9 Annoot et Fave-Bonnet (2004) pensent quant à elles qu'une réelle démocratisation de l'enseignement universitaire n'est possible seulement si des changements en matière de pédagogie interviennent, car les enseignants rencontrent souvent des difficultés pour 
mettre en œuvre des pratiques pertinentes. Paivandi (2012) rejoint d'ailleurs en quelque sorte cet avis puisqu'il considère que "pour éviter le décrochage précoce, intégrer et faire réussir les étudiants, l'université a besoin de réfléchir sur la pédagogie ».

\subsection{Des pratiques pédagogiques peu valorisées à l'université}

10 La multiplicité des tâches auxquelles sont confrontés les enseignants et le manque de valorisation de la fonction d'enseignement en raison d'une "prépondérance absolue de la recherche » (Bireaud, 1996) conduisent les universitaires à se sentir encore aujourd'hui tiraillés entre enseignement et recherche, comme avait déjà pu le démontrer Fave-Bonnet en 1994. Confrontés à cette diversité des fonctions qui leur sont assignées, et qui a par ailleurs conduit à la complexification de leur métier, il ne reste que peu de temps aux universitaires pour penser leurs pratiques pédagogiques, favorisant un certain " immobilisme pédagogique» (Bireaud, 1990). Leurs conceptions de l'enseignement sont d'ailleurs variées, et détermineraient en partie leurs pratiques pédagogiques (Romainville 2000), mais nous reviendrons ultérieurement sur ce fait. Altet (2004) met également en avant les problèmes pédagogiques rencontrés par les enseignants dans l'exercice de leur métier et auxquels ils doivent faire face, tels que les tensions disciplinaires, organisationnelles, celles provenant des attentes des étudiants ou encore celles dues au statut du métier d'enseignant-chercheur.

11 Outre la multiplicité des tâches confiées aux enseignants dans le supérieur, le peu de formation pédagogique dont ils bénéficient ne les incite pas à s'attarder sur leurs pratiques.

12 Ainsi, Adagnikou et Paul (2008) constatent que les centres de soutien à l'enseignement ne sont présents qu'au sein d'un peu plus de $20 \%$ des universités françaises. Parmi ces centres figurent les "Structures Universitaires de Pédagogie"(SUP). Celles-ci ont notamment pour vocation de "promouvoir les innovations pédagogiques", d' "assurer la formation initiale et continue des enseignants du supérieur", ou encore de "favoriser la valorisation de la fonction enseignante et de l'investissement pédagogique $»^{2}$. Mais de telles structures sont encore rares en France. Adagnikou et Paul (2008) mentionnent de surcroît le fait que finalement, seuls peu de centres proposent aux enseignants «des activités de formation et ou de conseil pédagogique » qui dépassent le seul usage des TICE (Adangnikou et Paul, 2008). Cependant, les missions qui leur sont dévolues témoignent tout de même d'une certaine préoccupation pour les pratiques enseignantes. Il s'agit là d'ailleurs d'un thème de plus en plus développé par la communauté scientifique, que cela soit en France ou en dehors de nos frontières. En témoigne la situation au Québec, où malgré la dévalorisation dont est victime l'enseignement, la recherche portant sur le sujet y est en véritable expansion, comme en atteste la présence dans chacune des universités d'un service de pédagogie universitaire. Cela prouve par ailleurs que cette dimension du métier d'enseignant-chercheur demeure au cœur des préoccupations, et que les universités s'intéressent véritablement à la problématique de l'enseignement universitaire (Boulet, Savoie-Zajc et Chevrier, 1996).

13 D'autres travaux, belges par exemple (Wouters, Frenay et Parmentier, 2011), portent sur un autre type de dispositif destiné à revaloriser les pratiques enseignantes à l'université : la mise en place du «Dossier de valorisation pédagogique». En effet, rédigé par les enseignants eux-mêmes, tout au long de leur carrière académique, et perçu de façon plutôt positive par ceux-ci, ce dispositif permet non seulement d'après les auteurs de 
mettre en œuvre "une forme d'évaluation de la qualité des pratiques pédagogiques de l'enseignant», mais contribue également largement au "développement chez l'enseignant d'une réflexion sur ses pratiques d'enseignement ».

\section{2. ... à l'analyse de l'impact de ces pratiques dans le supérieur}

14 La qualité des pratiques pédagogiques semble être un facteur important à ne pas négliger, puisque selon Annoot et Fave-Bonnet (2004), les actions de l'enseignant ne seraient par ailleurs pas "neutres" auprès des étudiants. En effet, pour nombre de chercheurs, les pratiques pédagogiques des enseignants ne sont pas sans conséquence sur les étudiants. Aussi Duru-Bellat (1995) alerte-t-elle quant au fait qu'il est important de ne pas négliger « les causes d'échec inhérentes au fonctionnement même de l'université ». D'ailleurs, si d'après Galinon-Mélénec (1996) il est clairement établi que l'enseignant et ses comportements jouent un rôle fondamental sur l'étudiant, pour Michaut (2012) en revanche les effets des pratiques pédagogiques, rarement abordés dans la littérature scientifique, constitueraient en réalité une véritable "zone d'ombre». Certaines recherches se sont ainsi penchées d'une part sur le ressenti des étudiants face aux pratiques pédagogiques universitaires, et sur leur impact réel sur les apprentissages de ces derniers d'autre part.

\subsection{La perception par les étudiants des différentes pratiques pédagogiques à l'université}

Dans un article publié en 2002, Soulié fait le point sur la perception, la satisfaction qu'ont les étudiants quant à leur formation universitaire, ainsi que sur leurs demandes pédagogiques éventuelles. Il en résulte que les étudiants mécontents réclament le plus souvent un changement de pratiques pédagogiques de la part de leurs professeurs. Ces derniers pourraient à titre d'exemple pour "manifester leur bonne volonté » épeler ou écrire les mots compliqués, dénier le rapport hiérarchique existant, ou encore proposer des plans de cours et polycopiés.

D'autres chercheurs se sont intéressés au ressenti des étudiants face aux pratiques pédagogiques de leurs professeurs. Ainsi, d'après Bru (2004), les étudiants «n'ont pas un jugement massivement négatif sur la façon dont l'enseignement universitaire est dispensé ». Cependant, "il leur arrive de regretter certaines formes d'enseignement». Pour certains étudiants, la première année universitaire serait même caractérisée par un "flou pédagogique » (Oberti, 1995) : le monde enseignant, comme il est bien souvent qualifié, est perçu par les nouveaux arrivants à l'université comme étant une "nébuleuse », dont ils ne perçoivent pas toujours « la logique ni l'organisation » (Felouzis, 2001).

17 Ainsi, les étudiants déplorent entre autre la «distance physique et morale » (Erlich, 1998) qui s'institue entre eux et les enseignants. Nombreux sont ceux à éprouver un "sentiment de délaissement » à leur entrée à l'université (Felouzis, 2001). Certains étudiants se sentent même méprisés par leurs enseignants. Comme l'explique Felouzis (2001), ils ont «le sentiment que le rapport pédagogique est en quelque sorte rompu par des attitudes qui dévalorisent les étudiants». Ce chercheur emploie même le terme de "mépris institutionnalisé » pour qualifier certaines situations, comme il peut en être le cas lors de la réunion de rentrée en filière de droit. Lors de cette assemblée, les étudiants sont bien souvent avertis du fait que 
seul l'un sur quatre d'entre eux parviendra à la réussite, les autres étant voués au redoublement ou à la réorientation (Felouzis, 2001). Ces propos sont confirmés par Altet, Fabre et Rayou (1999) qui rapportent le témoignage d'une étudiante inscrite en deuxième année de lettres modernes, et selon laquelle les enseignants mettent au point des " stratégies de découragement pour éliminer les étudiants peu performants et garder les meilleurs ". On peut alors aisément supposer qu'un tel discours de la part des enseignants risque de rapidement anéantir la motivation des étudiants, cela d'autant plus si cette dernière était déjà moindre à l'origine.

Cependant, malgré cet état de cause, il apparaît que les étudiants ont fréquemment tendance à avoir des avis quelque peu contradictoires à l'égard de leurs enseignants : malgré leur impression de ne pas être traités correctement, ils éprouvent tout de même un sentiment d'admiration pour leurs enseignants, la culture de ces derniers, ou encore le brio de leur carrière professionnelle. Finalement, comme l'exprime très bien Felouzis (2001), le "monde des "profs»" est un monde "étrange» pour les étudiants. Ceux-ci éprouvent de véritables difficultés à en saisir la véritable logique et l'organisation. Ils en parlent «comme d'un monde à part dont ils ne connaissent pas vraiment l'organisation hiérarchique ni le fonctionnement réel ». Or il est fort probable que cet inconnu et cette hostilité ne contribuent pas vraiment à un accroissement, ou tout au moins un maintien de la motivation des étudiants.

Bédard et Viau (2001) ont pour leur part cherché à identifier la façon dont sont perçues les méthodes d'enseignement des professeurs par les étudiants de l'université de Sherbrooke. Ainsi, ils ont demandé aux étudiants d'exprimer leur avis concernant six " activités», autrement dit des "situations d'enseignement»: l'exposé délivré par l'enseignant, les ateliers ${ }^{3}$, l'approche par problème ${ }^{4}$, l'approche par projet ${ }^{5}$, l'étude de cas $^{6}$ , et les séminaires de lecture ${ }^{7}$. Il s'avère que l'approche par projet est celle qui motive le plus les étudiants de première année, de même que l'étude de cas et l'approche par problème. En réalité, les étudiants n'éprouvent aucune difficulté à percevoir l'utilité de ces activités. Les auteurs précisent également que les étudiants se sentent plus compétents pour accomplir ces activités, et ont une meilleure perception de la contrôlabilité de leur déroulement. Ces "méthodes", comme on pourrait les nommer, dites généralement «actives» semblent donc être les mieux adaptées pour favoriser la motivation de l'étudiant. Il en est tout autrement en ce qui concerne l'exposé. Cette forme d'enseignement fait en réalité référence au système traditionnel : l'enseignant dispense ses savoirs, fait un exposé, et les étudiants écoutent, généralement en prenant des notes. Or pour les étudiants de premier cycle, cette forme d'enseignement n'est pas perçue comme étant très « utile ", ils n'ont pas «le sentiment d'avoir un contrôle sur son déroulement ", et il s'agit de "situations dans lesquelles les étudiants n'ont pas le sentiment qu'ils sont capables d'apprendre ». Or, si l'on se réfère à la dynamique motivationnelle de Viau (1998), ces éléments constituent des facteurs essentiels à la motivation des étudiants. Le type de cours prodigué, et donc par là même les pratiques pédagogiques employées en ce sens ne seraient donc pas sans effet sur la motivation des étudiants, facteur jouant un rôle essentiel dans les apprentissages. D'ailleurs, pour Viau (2006), il est «important pour les professeurs d'université de réfléchir sur les activités pédagogiques qu'ils proposent à leurs étudiants et de se demander dans quelle mesure elles contribuent à maintenir leur motivation tout au long de leurs études. " 


\subsection{Quel impact de ces pratiques sur les étudiants ?}

20 L'impact joué par les pratiques pédagogiques des enseignants sur la réussite des étudiants est depuis peu au cœur de questionnements. Galand, Neuville et Frenay (2005) émettent l'hypothèse qu'il est bien réel: aucune catégorie de facteurs habituellement passés en revue dans les différents travaux de recherche ne parvenant à expliquer dans son intégralité le phénomène échec, ils émettent la supposition que ce dernier pourrait bien être lié aux pratiques d'enseignement, et que les professeurs auraient donc bien un rôle à jouer. Romainville et Parmentier (1998) ajoutent que «les procédures que les étudiants déclarent mettre en cuvre à l'occasion d'un cours sont influencées par la méthode pédagogique de l'enseignant». Pour Romainville (2000), l'amélioration de la pédagogie à l'université pourrait même constituer une véritable solution pour lutter contre l'échec: «privilégier les méthodes qui suscitent l'apprentissage en profondeur ", « revaloriser la mission d'enseignement " ou encore " assurer des formations pédagogiques initiales ou continues " pourraient être des moyens de favoriser à terme la réussite des étudiants en premier cycle. Cette hypothèse était déjà avancée par Leroux (1997) qui décrit la pratique du cours magistral comme étant « inscrite dans la tradition universitaire » et constituant « un facteur d'échec en premier cycle».

21 De la même façon, des recherches au Québec se sont penchées sur l'impact des pratiques enseignantes sur les étudiants. Ainsi, Fontaine et Peters (2012) ont identifié la pédagogie des professeurs universitaires comme étant un facteur de "rétention», une pédagogie de qualité ayant une influence sur la persévérance des étudiants. Pageau et Médaille (2005) font le même constat, en ajoutant même que «le développement d'études en pédagogie universitaire pourrait contribuer à la réussite des étudiants». Ménard (2012) va plus loin en signifiant que les activités d'enseignement peuvent avoir un impact négatif sur l'apprentissage, si l'enseignant n'a pas «d'habiletés pédagogiques » et s'il n'est pas « concret ». Pour elle, les stratégies pédagogiques utilisées en classe sont importantes pour l'apprentissage et «tous les enseignants ne les maitrisent pas ». D'autres chercheurs anglosaxons ont pu montrer, tout comme les québécois, que les habiletés pédagogiques des enseignants avaient un impact positif sur l'apprentissage des étudiants, leur réussite scolaire, leur intégration sociale et leur engagement à poursuivre des études (Braxton, Bray et Berger, 2000).

De plus, est régulièrement mis en évidence le fait que ces pratiques pédagogiques pourraient avoir un effet indirect sur la réussite, en influençant les manières d'étudier des étudiants et leur motivation, conditionnant par là même leur réussite. Ainsi ceux évaluant positivement la qualité de l'enseignement qu'ils reçoivent s'investiraient même davantage dans leur travail académique. Le chercheur belge Wolfs (2007) rejoint cet avis dans son ouvrage " Méthode de travail et stratégies d'apprentissage " : après avoir mis à jour les différents types d'approches pédagogiques des enseignants, il dresse une liste des pratiques pédagogiques « visant à développer l'apprentissage en profondeur et le développement d'une réflexion métacognitive», sur laquelle figurent entre autres "l'utilisation de supports ouverts ", "d'outils de référence », ou encore de " méthodes incitatives ».

De maintes recherches restent encore à mener pour véritablement élucider le rôle des pratiques enseignantes à l'université. Ainsi, dans la conclusion de leur ouvrage « Réussite, échec et abandon dans l'enseignement supérieur ", regroupant des contributions de chercheurs français, belges, suisses, ou encore québécois, Romainville et Michaut (2012) 
estiment que « un axe à développer concerne les pratiques enseignantes elles-mêmes et leur rôle dans la promotion de la réussite ». En effet, si la pédagogie universitaire est couramment abordée, rares sont les travaux s'intéressant véritablement aux pratiques enseignantes, et à l'impact qu'elles peuvent avoir sur les étudiants, ce notamment en premier cycle universitaire. Le passage en revue de la littérature produite sur le sujet n'a permis de trouver que très peu d'écrits produits sur le sujet durant cette dernière décennie, d'où le fait d'ailleurs que les références citées dans cet article soient relativement anciennes. Pourtant, Romainville et Michaut (2012) estiment que «Echec et réussite apparaissent comme étant aussi sous l'emprise de l'action des enseignants, non pas dans une perspective culpabilisante sous entendant qu'ils sont à la source de l'échec, mais en indiquant que la qualité de leurs pratiques pédagogiques peut être déterminante dans la promotion de leur réussite, à exigences constantes ».

Les pratiques enseignantes ne semblent donc pas a priori être sans conséquence sur la scolarité des étudiants. Néanmoins, nous n'avons pu faire état d'aucune recherche empirique démontrant véritablement l'efficacité des pratiques de façon précise, ce notamment, comme nous l'avons évoqué précédemment, en raison d'un amalgame constant concernant la réelle signification du terme « pratiques pédagogiques ».

\section{Présentation d'une perspective de recherche novatrice sur le sujet}

En France, d'après le ministère de la recherche et de l'enseignement supérieur en 2007, plus d'un étudiant sur deux « échoue » en première année universitaire : $30 \%$ redoublent, $16 \%$ se réorientent et $6 \%$ abandonnent. Certes la définition de l'échec à proprement parlé demande, de l'avis de nombre de chercheurs, à être approfondie. D'autres tentent de relativiser la situation en précisant que si les taux d'échec ne diminuent pas, ils se sont au moins stabilisés (Nils \& Lambert, 2011). Mais le constat est tout de même là. Par conséquent, certaines politiques ont été développées pour tenter de combattre cet échec. Ainsi, on a vu apparaître en 2008 le Plan Réussite en Licence (PRL). Mais malgré ce plan visant à réduire de façon massive l'échec en premier cycle universitaire, le problème reste d'actualité. En effet, d'après un rapport produit par l'inspection générale de l'administration de l'éducation nationale (Bétant, Foucault \& Peyroux, 2010), les effets du PRL restent assez limités. Sa mise en place s'avère très aléatoire selon les universités et selon les filières. Les auteurs de ce rapport suggèrent par ailleurs de véritablement connaître les étudiants en situation d'échec pour parvenir à remédier à ce problème. La mise à jour des divers facteurs caractéristiques des étudiants dits "à succès " (Boulet et al., 1996), aussi appelés "déterminants de la réussite », est justement une tâche que les chercheurs se sont assignés depuis de nombreuses années. Les travaux de recherche consacrés à ce sujet ont majoritairement abordé l'impact des caractéristiques individuelles des étudiants sur leur réussite: passé scolaire, caractéristiques socio démographiques, conditions de vie, «métier d'étudiant » (Duru Bellat, 1995 ; Michaut, 2000 ; Romainville, 2000), motivation (De Ketele, 1990) ou encore plus récemment les capacités cognitives (Morlaix \& Suchaut, 2012). Bien que plus rares, d'autres travaux se sont penchés sur le rôle joué par le contexte universitaire sur la réussite des étudiants (Michaut, 2000). 
26 Mais tous ces facteurs ne suffisent pas à expliquer le phénomène "échec » dans son intégralité : les études intégratives parviennent à en expliquer $25 \%$ au maximum (Robbins et al., 2004), et dans tous les cas jamais plus de 50 \% (Galand, Neuville et Frenay, 2005). Certes il existe une part d'éléments inhérents à l'étudiant que l'on ne pourra jamais expliquer. Mais aucune recherche ne s'est jusqu'à présent véritablement intéressée au rôle joué par l'enseignant et ses pratiques. Certes certaines "études courageuses" (Romainville, 2005) ont tenté de montrer que les pratiques pédagogiques des enseignants n'étaient pas sans effets sur les apprentissages des étudiants. Mais ces travaux restent rares. Le sujet est généralement tabou car cela «reviendrait pour les chercheurs à se mettre en jeu, à s'exposer à la critique» (Romainville, 2005). Il faut ajouter à cela que les enseignants ont dans la globalité une opinion plutôt négative des étudiants débutants (Boyer \& Coridian, 2001). Bien souvent, ils considèrent ceux-ci comme étant une « masse uniforme» (Mucchielli, 1998), et dénoncent les lacunes des étudiants: manque de méthodes du travail intellectuel, manque de travail et d'organisation, maîtrise insuffisante de l'expression écrite, orale et de l'orthographe (Boyer et Coridian, 2001). Ainsi sont-ils nombreux à préférer rejeter la faute de l'échec sur l'étudiant, se dédouanant ainsi de toute responsabilité (Romainville, 2000).

Comme nous l'avons déjà montré auparavant, certaines recherches laissent pourtant entendre que les pratiques enseignantes ne seraient elles aussi pas sans effet sur les étudiants. Par ailleurs, le passage en revue de la littérature scientifique existante sur le sujet nous a conduit à constater que l'université ne semblait plus à l'heure actuelle véritablement répondre aux besoins des étudiants. La question de l'efficacité des pratiques enseignantes se place donc aujourd'hui encore au cœur des débats.

C'est justement dans ce cadre que nous souhaitons présenter une perspective de recherche novatrice, actuellement développée à l'IREDU (Institut de Recherche en Éducation), et faisant actuellement l'objet d'une thèse de doctorat en cours de rédaction. Elle se propose d'aborder ce thème à travers deux dimensions : celle de la variété des pratiques enseignantes en cours magistral en première année universitaire d'une part, et d'autre part celle de leur efficacité, autrement dit de leurs effets sur la scolarité des étudiants.

\subsection{Des pratiques hétérogènes?}

Les pratiques des enseignants du supérieur vont varier en fonction d'un certain nombre de facteurs, le premier étant certainement la conception que l'enseignant a de sa mission d'enseignant-chercheur, et de la relation aux étudiants qu'il veut construire. Certains auteurs (Demougeot-Lebel \& Perret, 2010a, 2010b; Postareff \& Lindblom-Ylänne, 2011; Sadler, 2008; Trigwell, 2009) démontrent que ces conceptions varient également en fonction des caractéristiques personnelles des enseignants, et de leur expérience dans l'enseignement, ces différences de conceptions ayant au final un impact sur leurs pratiques pédagogiques. (Langevin et al., 2007 ; Trigwell \& Prosser, 2004). Comme nous le rappelle Lambert (2012), ces façons d'enseigner auront ensuite un poids sur l'apprentissage de l'étudiant et sa réussite. Il existerait ainsi un continuum de pratiques allant d'après Romainville " "du pourvoyeur" d'informations au facilitateur d'apprentissage " $(1998,2000)$. Kember (1997) classifie les conceptions de l'enseignement en cinq catégories caractérisant la nature de la mission des enseignants. Romainville (2000) rappelle cette classification des catégories. Ainsi, enseigner peut signifier " transmettre de l'information ", 
" transmettre un savoir ", " gérer des interactions professeur / étudiant ", " aider à apprendre » ou bien encore « favoriser un changement de conception » de l'étudiant.

Ces différences de conceptions de l'enseignement par l'enseignant vont influer sur les pratiques pédagogiques mises en place, ces pratiques pédagogiques étant considérées bien souvent comme étant l'affaire de l'enseignant seul et relevant d'une moindre importance (Lahire, 1997) comparativement aux autres tâches qui lui sont assignées. En effet, en France, le métier d'enseignant chercheur s'est à la fois diversifié et complexifié (Altet, 2004). Les enseignants se sont vus contraints, face à l'hétérogénéité grandissante de leur public, de faire évoluer les contenus, les orientations des formations (Dejean 2002), mais aussi leurs pratiques pédagogiques. Mais l'activité d'enseignement est bien peu valorisée par rapport à la recherche. Nous sommes partis de l'hypothèse que malgré la prédominance du modèle expositif, les pratiques enseignantes en cours magistral sont hétérogènes. Aussi avons-nous cherché à décrire et à catégoriser les pratiques pédagogiques individuelles d'enseignants exerçant au sein de filières dites « de masse ». Dans ce cadre, nous avons procédé à l'observation directe de cours magistraux dispensés par 49 enseignants dans les filières de droit, administration économique et sociale, sciences de la vie et de la terre, psychologie, sociologie et LLCE ${ }^{8}$ anglais. Les observateurs étaient à cet effet munis de grilles d'observation destinées à appréhender les pratiques pédagogiques des enseignants relatives à six dimension: la manière d'interagir de l'enseignant avec les étudiants, son utilisation du matériel, sa façon d'organiser le cours, sa clarté, sa façon de transmettre le cours et enfin son attitude

31 La phase d'observation venant de prendre fin, nous ne sommes pas véritablement encore en mesure de confirmer, infirmer ou nuancer notre hypothèse. Cependant, cette phase nous a semblé indispensable pour mener à bien un second objectif: celui d'analyser l'efficacité des pratiques enseignantes, cela à travers la mise à jour de leur impact sur la scolarité des étudiants. Or cet impact pourrait bien s'avérer triple, puisqu'il pourrait s'exercer à la fois sur la motivation des étudiants, leurs manières d'étudier, mais aussi sur leur réussite en termes de résultats aux examens.

\subsection{Tester l'impact de ces pratiques sur la motivation des étudiants et leurs manières d'étudier}

Beaucoup de recherches ont analysé la relation entre motivation et performance scolaire, comme il en est le cas de Lambert-Le Mener (2012). Cette dernière rappelle dans ses travaux qu'une des manières d'augmenter la motivation dans le milieu scolaire passe souvent par l'augmentation de la motivation extrinsèque, plus aisément modifiable pour un acteur extérieur, et notamment par les enseignants.

Par ailleurs, la littérature rédigée sur le sujet fait état de pratiques enseignantes qui ne seraient pas sans effet sur la motivation des étudiants de première année. C'est en tout cas ce que mettent en évidence Boyer, Coridian et Erlich (2001). D'après ces derniers, nombre d'enseignants «ne se préoccupent pas vraiment d'accrocher l'attention de leur auditoire». Pourtant, pour une partie des étudiants de L1, "l'intérêt pour telle ou telle matière a encore du mal à exister en dehors de la personne de l'enseignant » (Barrère 1997, citée par Erlich, 1998). On en déduit donc qu'une pratique de transmission des savoirs inadaptée exerce un impact sur la motivation des étudiants puisque ceux-ci auront sans doute plus tendance à "décrocher » s'ils peinent à suivre et comprendre le contenu du cours, ou si cela leur paraît inintéressant. Cette hypothèse est confirmée par Erlich (1998), 
selon qui les étudiants « se sentent mal encadrés », et " mal assistés » par les enseignants. Or, d'après cette chercheuse, cette situation aurait pour conséquence une faible motivation des étudiants dans leur travail. Rappelons ainsi que Soulie (2002), après avoir invité des étudiants en première année de sociologie à évaluer leur formation, a constaté que les plus mécontents vis-à-vis de leur formation avaient deux fois plus souvent que les autres réclamé un changement de pratiques pédagogiques de la part de leurs enseignants. Ils ont notamment émis le souhait de voir leurs enseignants ralentir, répéter et même dicter plus souvent le cours. Notons par ailleurs que, d'après Sénécal et al. (1992) ${ }^{9}$, les pédagogies dites actives seraient plus propices au développement d'une motivation intrinsèque chez les étudiants (Harter, 1981) ${ }^{10}$, tandis que Solomon et Kendall $(1976)^{11}$ estiment, eux, que la pédagogie dite "traditionnelle», et donc par la même les pratiques qualifiées de «contrôlantes » généralement employées par les enseignants durant les cours magistraux, conduisent au développement d'une motivation extrinsèque. Certes Ryan et Grolnick (1986) ${ }^{12}$ avertissent quant au fait que "ces différences motivationnelles sont plus associées à la perception que les sujets ont de l'enseignant qu'à la réalité de ses pratiques pédagogiques ", les pratiques enseignantes n'en paraissent pas moins avoir tout de même un effet non négligeable sur la motivation des étudiants. D’après ces divers constats, la motivation des étudiants, au sens où l'entend Viau (1998), c'est-à-dire comme étant «un état dynamique qui a ses origines dans les perceptions qu'un élève à de lui-même et de son environnement et qui l'incite à choisir une activité, à s'y engager et à persévérer dans son accomplissement afin d'atteindre un but ", ne serait donc pas un phénomène indépendant des pratiques enseignantes.

Il apparaît donc envisageable de mesurer et de quantifier le poids que peuvent jouer ces pratiques sur la motivation des nouveaux arrivants à l'université.

Dans un deuxième temps, nous avons pu constater à travers nos lectures que la réussite aux examens et le passage à l'année supérieure, étaient dépendants des manières d'étudier des étudiants (Felouzis, 2003), ce que Michaut (2000) nomme autrement « l'implication dans les études». Lahire (1997), dans son ouvrage justement intitulé «les manières d'étudier" s'est attaché à décrire l'ensemble des différentes dimensions constituant les manières d'étudier afin de mettre à jour leur variété en fonction des diverses filières d'inscription des étudiants. C'est ainsi qu'il s'est notamment intéressé aux «rythmes de travail universitaires", à "l'organisation de l'emploi du temps", à la fréquentation des lieux universitaires par les étudiants, à leurs pratiques de lecture ou encore aux "conditions matérielles de travail scolaire». Par ailleurs, lorsqu'est fait un inventaire des différentes recherches produites sur le thème des manières d'étudier, ou des «manières d'apprendre ", terme préféré par Parmentier et Romainville (1998), on constate que celles-ci ne sont pas sans entretenir d'étroites relations avec les «stratégies d'apprentissage ». Ce concept de "manières d'étudier » comprend donc, dans le cadre où nous l'employons ici, à la fois les pratiques employées par les étudiants pour étudier et réviser durant leur temps libre et les cours, puisque même à ces instants tous n'adoptent pas les mêmes "pratiques informelles d'étude " (Alava, 2000), mais aussi les stratégies d'apprentissage mises en œuvre par les étudiants, que celles-ci soient d'ordre cognitives, affectives, métacognitives ou bien encore de gestion des ressources. On connait déjà le fait que les manières d'étudier soient un facteur de discrimination et de réussite (Lahire, 1997 cité par Alava, 2000). Certaines façons d'étudier sont plus propices à la réussite que d'autres: assiduité aux cours, travail régulier, planification d'un emploi du temps, détermination de stratégies adaptées à chaque cours, gestion de l'équilibre entre le 
travail et les loisirs (Rey, 2005). Mais il semble que les manières d'étudier soient elles aussi en partie liées aux pratiques pédagogiques des enseignants. En effet, comme le mentionne Endrizzi en 2010 dans une recension de la littérature, des exigences pédagogiques différentes conduisent les étudiants à des manières d'étudier très différentes. Ainsi, à titre d'exemple, Romainville et Parmentier (1998) évoquent le fait que «les procédures que les étudiants déclarent mettre en cuvre à l'occasion d'un cours sont influencées par la méthode pédagogique de l'enseignant ». D'ailleurs, rappelons que pour Ramsden (1988), l'enseignement fait partie des variables contextuelles influençant les manières d'apprendre de l'étudiant. Ces constats conduisent aux interrogations suivantes: Quelle est l'implication des pratiques pédagogiques des enseignants dans la motivation et l'organisation dans le travail des étudiants? Certaines pratiques sont-elles plus motivantes que d'autres?

Enfin, les précédentes recherches menées sur le sujet ayant postulé un effet des pratiques enseignantes sur la motivation ainsi que sur les manières d'étudier, et ces deux facteurs jouant eux-mêmes un rôle sur la réussite, il semble possible de pouvoir mettre à jour un impact, direct et indirect, des pratiques pédagogiques des enseignants sur les étudiants de $1^{\text {ère }}$ année universitaire en termes de réussite aux examens, et de comparer cet impact à celui d'autres variables, dont on sait déjà qu'elles jouent sur la réussite des étudiants, comme le passé scolaire et le métier d'étudiant.

Les réponses fournies par 734 étudiants à une enquête par questionnaire abordant plusieurs dimensions, telles que celles relatives à leurs caractéristiques sociodémographiques, leur passé scolaire, leur(s) motivation(s), leurs manières d'étudier, ainsi que leur opinion concernant les pratiques pédagogiques employées par leurs enseignants, seront mises en rapport avec les pratiques enseignantes observées, et les résultats des étudiants aux examens ${ }^{13}$. Ce travail empirique étant en cours, nous n'avons encore pu élaborer de premiers résultats. Cependant, il nous paraît important de mentionner qu'outre la création de modèles de régression multiples destinés à comparer "toutes choses égales par ailleurs " l'impact des différents facteurs sur la réussite des étudiants, les résultats pourront, et c'est là encore que la recherche est véritablement novatrice, être analysés à l'aide du logiciel LISREL offrant la possibilité de construire des modèles structurels et ainsi de considérer l'ensemble de ces facteurs, autrement dit la motivation, les manières d'étudier, la réussite et les pratiques pédagogiques, comme étant des concepts latent, c'est à dire des variables non observables et donc traduites par un certain nombre d'indicateurs. Ce type d'analyse permettra de "spécifier les relations" entre ces différents facteurs et de "préciser le type de relation envisagée entre les variables latentes » (Morlaix, 2002).

Enfin, il est important de préciser que cette recherche, à visée heuristique, n'a en aucun cas pour but de dénoncer telle ou telle pratique comme étant bonne ou mauvaise. L'objectif est plutôt de mettre à jour la variété des pratiques et de voir comment celles-ci jouent sur les étudiants, s'il s'agit d'un élément permettant d'expliquer une grand part de la variabilité des différences de résultats entre ces derniers, et donc s'il est réellement nécessaire d'essayer d'agir sur ces pratiques, en trouvant des leviers permettant d'améliorer la formation pédagogique des personnels universitaires par exemple, pour favoriser la réussite des nouveaux arrivants à l'université. Une autre visée de ce travail empirique s'inscrivant, de même que la majorité des travaux cités dans cet article, dans le cadre du paradigme sociologique des processus-produit est par ailleurs d'aboutir à une 
certaine théorisation pour contribuer à combler les manques actuels dans la littérature sur ce thème.

\section{Conclusion}

Même si les pratiques enseignantes en première universitaire ont fait l'objet d'un certain nombre de recherches françaises ou étrangères, la question de leur variété, mais aussi et surtout de leur efficacité reste aujourd'hui encore peu documentée. Pourtant, nombre d'étudiants semblent encore actuellement rencontrer des difficultés face aux pratiques de leurs enseignants, cela surtout lors des cours magistraux. Or, même si la tendance reste portée sur le modèle expositif, il existe tout de même, au moins d'après Clanet (2001), une certaine variété des pratiques. Or, nous n'avons pu faire état d'aucune recherche s'étant véritablement attachée à décrire et à catégoriser l'hétérogénéité des pratiques en cours magistral, et à mesurer précisément l'impact que celles-ci sont susceptibles d'avoir sur la scolarité des étudiants. Pourtant, comme nous l'avons vu, celles-ci ne seraient pas sans effet sur la motivation des étudiants, sur leurs manières d'étudier ainsi que sur leur réussite en termes de résultats aux examens. Le développement de travaux tels que la recherche innovante présentée précédemment, notamment dans sa perspective de quantifier l'effet des pratiques sur les étudiants, apparait donc comme une véritable nécessité, dans un contexte où les recherches portant sur la pédagogie universitaire sont en pleine expansion, et où l'échec massif en première année universitaire reste au cœur des débats. Un tel travail permettra de surcroit de mettre à jour certaines pistes de réflexion concernant la formation pédagogique des enseignants, à l'heure où l'on prévoit l'ouverture pour la rentrée 2013 d'Écoles Supérieures du Professorat et de l'Éducation dévolues notamment à la formation à la pédagogie des enseignants se destinant à une carrière dans le primaire, le secondaire ou le supérieur.

\section{BIBLIOGRAPHIE}

Adangnikou, N. (2008). Peut-on parler de recherche en pédagogie universitaire, aujourd'hui, en France? Revue des Sciences de l'Éducation, 34(3), 601-621.

Adangnikou, N., \& Paul, J. J. (2008). La formation à la pédagogie universitaire en France, une offre encore trop restreinte. $25^{\circ}$ Congrès de l'Association Internationale de Pédagogie Universitaire. 19-22 mai, Montpellier.

Alava, S. (2000). Les profils d'auto-direction et les pratiques d'études des étudiants en première année d'université. Les Sciences de l'Éducation pour l'Ère Nouvelle, 33(1), 43-71.

Altet, M. (1994). Le cours magistral universitaire : un discours scientifico-pédagogique sans articulation enseignement-apprentissage. Recherche et Formation, 15, 35-44.

Altet, M. (2004). Enseigner en premier cycle universitaire : Des formes émergentes d'adaptation ou de la « métis » enseignante. In E. Annoot \& M.F. Fave Bonnet, Pratiques pédagogiques dans l'enseignement supérieur : enseigner, apprendre, évaluer (pp. 37-52). Paris : L'Harmattan. 
Annoot, E., \& Fave Bonnet, M. F. (2004). Pratiques pédagogiques dans l'enseignement supérieur : enseigner, apprendre, évaluer. Paris : L'Harmattan.

Beaud, S. (2002). $80 \%$ au bac ... et après ? Les enfants de la démocratisation scolaire. Paris : la Découverte.

Bédard, D., \& Viau, R. (2001). Le profil d'apprentissage des étudiantes et des étudiants de l'université de Sherbrooke. Résultats de l'enquête menée au trimestre d'automne 2000. Rapport au vice-rectorat à l'enseignement. Université de Sherbrooke.

Bétant, B., Foucault, M., \& Peyroux, C. (2010). Note relative à la mise en oeuvre du plan pour la réussite en licence. ( $\mathrm{N}^{\circ}$ 2010-091). Rapport de l'Inspection Générale de l'Administration de l'Education Nationale et de la Recherche.

Bireaud, A. (1990). Les méthodes pédagogiques dans l'enseignement supérieur. Paris : Ed. d'organisation.

Bireaud, A. (1996). En France, une politique de formation pédagogique pour les enseignants du supérieur timide, hésitante et controversée. In J. Donnay \& M. Romainville (Dir.) : Enseigner à l'Université : un métier qui s'apprend? (pp.113-122). Bruxelles : De Boeck Université.

Boulet, A., Savoie Zajc, L., \& Chevrier, J. (1996). Les stratégies d'apprentissage à l'université. SainteFoy : Presses de l'Université du Québec.

Bourdieu, P., \& Passeron, J. C. (1964). Les héritiers. Les étudiants et la culture. Coll. « Le sens commun ». Paris : Minuit.

Boyer, R., \& Coridian, C. (2002). Transmission des savoirs disciplinaires dans l'enseignement universitaire, une comparaison histoire-sociologie. Sociétés contemporaines, 48, 41-61.

Boyer, R., Coridian, C., \& Erlich, V. (2001). L'entrée dans la vie étudiante. Socialisation et apprentissages. Revue Française de Pédagogie, 136, 97-107.

Braxton, J. M., Bray, N. J., \& Berger, J. B. (2000). Faculty Teaching Skills and their Influence on the College Student Departure Process. Journal of College Student Development, 41(2), 215-227.

Bressoux, P. (1994). Les recherches sur les effets-écoles et les effets-maîtres. Revue Française de Pédagogie, $\mathrm{n}^{\circ}$ 108, 91-137.

Bressoux P. (2001). Réflexions sur l'effet-maître et l'étude des pratiques enseignantes, Les dossiers des sciences de l'éducation, $\mathrm{n}^{\circ}$ 5, p. 35-52.

Bru, M. (2004). Pratiques enseignantes à l'université : Opportunité et intérêt des recherches. In E. Annoot et M.F. Fave Bonnet : Pratiques pédagogiques dans l'enseignement supérieur : enseigner, apprendre, évaluer (p.17-36). Paris : L'Harmattan.

Bru, M. (2006). Les méthodes en pédagogie. Que sais-je. Paris : Presses Universitaires de France.

Clanet, J. (2001). Etude des organisateurs des pratiques enseignantes à l'université. Revue des Sciences de l'Education, vol. 27 ( $\left.\mathrm{n}^{\circ} 2\right)$, p. 327-352.

Coulon, A. (2005). Le métier d'étudiant : l'entrée dans la vie universitaire (2ème éd.). Paris :

Economica : Anthropos.

De Ketele, J. M. (1990). Le passage de l'enseignement secondaire à l'enseignement supérieur : les facteurs de réussite. Vie pédagogique, ( ${ }^{\circ}$ 66), p. 4-8.

Dejean, J. (2002). L'évaluation de l'enseignement dans les universités françaises. Rapport établi à la demande du Haut Conseil de l'évaluation de l'école (108p.). 
Demougeot-Lebel, J., \& Perret, C. (2010). Identifier les conceptions de l'enseignement et de l'apprentissage pour accompagner le développement professionnel des enseignants débutants à l'université. Savoirs, 2(23), 51-72.

Duru Bellat, M. (1995). Des tentatives de prédiction aux écueils de la prévention en matière d'échec en première année d'université. Savoir Éducation / Formation, vol. 3, 399-416.

Endrizzi, L. (2010). Réussir l'entrée dans l'enseignement supérieur. Dossier d'actualité INRP, Veille scientifique et technologique, ( $\left.{ }^{\circ} 59\right)$.

Erlich, V. (1998). Les nouveaux étudiants : un groupe social en mutation. Paris : Armand Colin.

Fave-Bonnet, M. F. (1994). Le métier d'enseignant chercheur : des missions contradictoires. Recherche et Formation, $\mathrm{n}^{\circ} 15,11-34$.

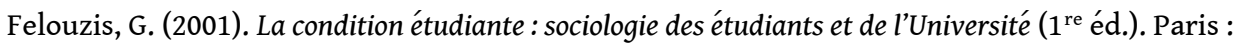
Presses universitaires de France.

Felouzis, G. (2003). Les mutations actuelles de l'Université (1re éd.). Paris : Presses Universitaires de France.

Fontaine, S., \& Peters, M. (2012). L'abandon des étudiants à l'université : Etat de la question. In M. Romainville \& C. Michaut : Réussite, échec et abandon dans l'enseignement supérieur, Coll. Perspectives en Education et Formation (pp. 33-52). Bruxelles : De Boeck.

Forner, Y., \& Simonot, C. (2001). Motivation et adaptation à l'université. Psychologie et psychométrie, 22(1), 59-73.

Galand, B., Neuville, S., \& Frenay, M. (2005). L'échec à l'université en Communauté Française de Belgique : Comprendre pour mieux prévenir ? In B. Galand (dir.) : L'échec à l'université en Communauté Française de Belgique (pp. 5-17). Cahiers de recherche en éducation et formation, $\mathrm{n}$ - 39.

Galinon-Mélénec, B. (1996). L'enseignant chercheur au sein d'une situation complexe et contingente. In J. Donnay et M. Romainville (Dir.) : Enseigner à l'Université : un métier qui s'apprend? (pp.11-31). Bruxelles : De Boeck Université.

Galland, O., \& Oberti, M. (1996). Les étudiants. Paris : Editions La Découverte. Jarousse, J. P. (1984). Les contradictions de l'université de masse 10 ans après. Revue Française de sociologie, 25(2), 191-210.

Kember, D. (1997). A reconceptualisation of the research into university academics conceptions of teaching. Learning and Instruction, 7(3), 255-275.

Lahire, B. (1997). Les manières d'étudier. Cahiers de l'OVE $n^{\circ} 2$. Paris : La documentation française.

Lambert, M. (2012). La performance académique des étudiants en première année universitaire : influence des capacités cognitives et de la motivation. Thèse de doctorat de l'Université de Bourgogne en sciences de l'éducation, novembre 2012.

Langevin, L., Bilodeau, H., Boisclair, M., \& Bracco, M.A. (2007). Formation et soutien à l'enseignement universitaire : Des constats et des exemples pour inspirer l'action. Sainte-Foy : Presses de l'université du Québec.

Leroux, J. Y. (1997). Les premiers cycles universitaires français dans le contexte de l'enseignement supérieur de masse. Gestion de l'enseignement supérieur, 9(1), 115-125.

Lévy-Garboua, L. (1976). Les demandes de l'étudiant ou les contradictions de l'université de masse. Revue française de sociologie, 1(17), 53-80. 
Postareff L. \& Lindblom-Ylänne S. (2011). Emotions and confidence within teaching in higher education. Studies in Higher Education, 36(5).

Ménard, L. (2012). Apprentissage en classe et persévérance au premier cycle universitaire. In M. Romainville et C. Michaut : Réussite, échec et abandon dans l'enseignement supérieur, Coll. Perspectives en Education et Formation (pp.177-198). Bruxelles : De Boeck.

Michaut, C. (2000). L'influence du contexte universitaire sur la réussite des étudiants. (Thèse de doctorat, sous la direction de Duru Bellat M.). Université de Bourgogne, Institut de recherche sur l'économie de l'éducation.

Michaut, C. (2012). Réussite, échec et abandon des études dans l'enseignement supérieur français : quarante ans de recherche. In M. Romainville et C. Michaut : Réussite, échec et abandon dans l'enseignement supérieur (pp. 53-68), Coll. Perspectives en Education et Formation. Bruxelles : De Boeck.

Morandi, F., \& La Borderie, R. (1998). Modèles et méthodes en pédagogie. Collection 128. Paris : Nathan.

Morlaix, S., \& Suchaut, B. (2012). Les déterminants scolaires, sociaux et cognitifs de la réussite en première année universitaire. Revue Française de Pédagogie, à paraitre.

Mucchielli, L. (1998). La pédagogie universitaire en question : Le point de vue des étudiants de premier cycle en psychologie. Recherche et formation, ( $\left.n^{\circ} 29\right), 161-176$.

Nils, F. \& Lambert, J.P. (2011). Quelle est l'ampleur de l'échec en première année à l'université ? Tout est-il joué d'avance? In Ph. Parmentier (Dir.). Recherches et actions en faveur de la réussite en première année universitaire. Vingt ans de collaboration dans la Commission « Réussite » du Conseil interuniversitaire de la Communauté française de Belgique (pp.10-13). Bruxelles : CIUF.

Oberti, M. (1995). Les étudiants et leurs études. In O. Galland (dir.) : Le monde des étudiants (pp. 23-54). Paris : Presses Universitaires de France.

Pageau, D., \& Médaille, C. (2005). La recherche institutionnelle au Québec. In P. Chenard \& P. Doray (dir.) : L'enjeu de la réussite dans l'enseignement supérieur (pp.111-126). Sainte-Foy : Presses de l’Université du Québec.

Paivandi, S. (2012). Pour réussir la transition entre secondaire et supérieur. Cahiers pédagogiques. Hors-série numérique $n^{\circ} 25$, "Quelle pédagogie dans le supérieur ? ", 22-25.

Peretti, C. (dir) (2009). Rapport sur les Centres d'Initiation à l'Enseignement Supérieur ( $\mathrm{N}^{\circ}$ 2009-055).

Prost, A. (1986). L'enseignement s'est-il démocratisé ? Paris : Presses Universitaires de France.

Ramsden, P. (1988). Improving learning: New perspective. Londres : Nichols Pub Co.

Rayou, P. (1999). Une secondarisation. In Altet M., Fabre M. et Rayou P. (dir.) : Hétérogénéité et réussite dans le premier cycle universitaire. Enseignants et étudiants. Représentations, pratiques et adaptation des métiers. Rapport de recherche, INRP/CNCRE. Paris.

Rey, O. (2005). L'enseignement supérieur sous le regard des chercheurs. Institut National de Recherche Pédagogique. Dossier de Veille Scientifique et Technologique.

Robbins, S. B., Lauver, K., Le, H., Davis, D., \& Langley, R. (2004). Do psychosocial and study skill factors predict college outcomes ? A meta-analysis. Psychological Bulletin, ( $\left.n^{\circ} 130\right), 261-288$.

Romainville, M. (2000). L'échec dans l'université de masse. Paris : L'Harmattan. 
Romainville, M. (2005). Quelques interrogations sur l'échec à l'université. In B. Galand (dir.) : L'échec à l'université en Communauté Française de Belgique (pp.18-22). Cahiers de recherche en éducation et formation, $\mathrm{n}^{\circ} 39$.

Romainville, M., \& Michaut, C. (dir). (2012). Réussite, échec et abandon dans l'enseignement supérieur. Perspectives en éducation et formation. Bruxelles : De Boeck Université.

Romainville, M., \& Parmentier, P. (1998). Les manières d'apprendre à l'université. In M. Frenay, B. Noël, P. Parmentier, et M. Romainville (dir.) : L'étudiant-apprenant : Grilles de lecture pour l'enseignant universitaire (pp. 63-80). Bruxelles: De Boeck Université.

Sadler, I. (2008). Development of new teachers in higher education: Interactions with students and other influences upon approach to teaching. PhD thesis. University of Edinburgh.

Soulie, C. (2002). L'adaptation aux « nouveaux publics » de l'enseignement supérieur : Autoanalyse d'une pratique d'enseignement magistral en sociologie. Sociétés contemporaines, 4(48), 11-39.

Trigwell, K. (2009). Relations between teachers' emotions in teaching and their approaches to teaching in higher education: A pilot study. In EARLI 2009, Amsterdam, 25-29 août 2009.

Trigwell, K. \& Prosser, M. (2004). Development and Use of the Approaches to Teaching Inventory. Educational Psychology Review, 16(4), 409-424.

Trinquier, M. P., Clanet, J., \& Alava, S. (1999). Hétérogénéité et réussite dans le premier cycle universitaire. Conditions perçues et effectives des pratiques d'études et d'enseignement. Rapport de recherche, INRP/CNCRE. Paris.

Vasconcellos, M. (2006). L'enseignement supérieur en France. Paris : la Découverte.

Viau, R. (1998). La motivation en contexte scolaire. ( $2^{\mathrm{e}}$ éd.). Bruxelles : De Boeck.

Viau, R. (2006). La motivation des étudiants à l'université : mieux comprendre pour mieux agir. Conférence non publiée. Université de Liège.

Wolfs, J.-L. (2007). Méthodes de travail et stratégies d'apprentissage : du secondaire à l'université : Recherche, théorie, application ( $3^{\text {e }}$ éd.). Bruxelles : De Boeck.

Wouters, P., Frenay, M., \& Parmentier, P. (2011). Valoriser l'engagement pédagogique des enseignants chercheurs. Recherche et Formation : Former les universitaires en pédagogie. Dossier coordonné par Frenay M. et Paquay L., (nº 67), 73-90.

\section{NOTES}

1. Seront principalement passés en revue les travaux produits par les chercheurs français, mais aussi belges et québécois, cela pour plusieurs raisons : leurs modes de fonctionnement sont proches de celui de la France, et ces chercheurs travaillent régulièrement en interaction avec les chercheurs de l'hexagone. Les recherches produites par les auteurs anglo-saxons ne seront que très peu évoquées : elles sont rares, peu sont récentes, et elles abordent la pédagogie au sens général, elles ne se focalisent pas véritablement sur les pratiques pédagogiques en tant que telles. 2. Texte fondateur du réseau des SUP, Juin 2010.

3. "Réalisations en équipe d'exercices ou de travaux portant sur les notions étudiées pendant le cours » (Bédard \& Viau, 2001).

4. "Recherche de notions théoriques nécessaires à la compréhension d'un problème d'envergure et éventuellement à sa résolution » (Bédard \& Viau, 2001). 
5. «Réalisation d'un projet d'équipe qui comporte les mêmes étapes et les mêmes contraintes que l'on retrouve dans la vie professionnelle » (Bédard \& Viau, 2001).

6. "Situations se rapprochant de la réalité, dans lesquelles l'étudiant doit analyser la situation sous tous ses angles afin de choisir des solutions nuancées » (Bédard \& Viau, 2001).

7. «Préparation d'un compte rendu de lectures et présentation à des collègues » (Bédard \& Viau, 2001).

8. Langue, lettres et civilisation étrangère

9. Cités par Forner et Simonot (2001)

10. Cité par Forner et Simonot (2001)

11. Dont les propos sont repris par Forner et Simonot (2001)

12. Cités par Forner et Simonot, (2001)

13. Ces résultats seront obtenus grâce à un accès à la base de données APOGEE.

\section{RÉSUMÉS}

En France, certains travaux (Boyer \& Coridian, 2002; Clanet, 2001) ont mis en lumière l'hétérogénéité des pratiques enseignantes à l'université, notamment en première année, sans toutefois analyser, de façon précise, les effets de cette variété sur les étudiants. Pourtant, ces pratiques ne seraient pas sans conséquence sur ces derniers (Bireaud, 1990 ; Duru Bellat, 1995 ; Galinon Menelec, 1996). C'est donc à cette réflexion qu'est dédiée la recherche présentée dans cet article. Dans un premier temps seront recensés les travaux produits en France et à l'international sur la description des pratiques. La seconde partie portera sur les effets induits par celles-ci sur les étudiants. Enfin, sera évoquée une perspective de recherche novatrice, portant sur la relation entre pratiques pédagogiques des enseignants et motivation ainsi que manières d'étudier des étudiants de 1ère année universitaire.

In France, some studies (Boyer \& Coridian, 2002; Clanet, 2001) have highlighted the heterogeneity of teaching practices at the university, especially in the first year, but don't analyze accurately the effects of this variety on the students. However, these practices are not without consequences for them (Bireaud 1990; Bellat Duru, 1995; Galinon Menelec, 1996). So it is to this reflection that is dedicated the research presented in this article. Firstly will be presented studies in France and abroad about the description of practices. The second part will focus on the effects induced by them on students. Finally, we will present an innovative viewpoint of research, on the relationship between teaching practices and motivation as well as ways of studying students first academic year.

\section{INDEX}

Keywords : teaching practices in university, freshman year, heterogeneity and efficiency practices, motivation, ways of studying

Mots-clés : pratiques pédagogiques à l'université, première année universitaire, hétérogénéité et efficacité des pratiques, manières d'étudier 


\section{AUTEURS}

AMÉLIE DUGUET

IREDU/CNRS-Université de Bourgogne

SOPHIE MORLAIX

IREDU/CNRS-Université de Bourgogne 